\title{
HyPro: A Multi-DoF Hybrid-Powered Transradial Robotic Prosthesis
}

\author{
C. L. Semasinghe $(\mathbb{D}$, R. K. P. S. Ranaweera, J. L. B. Prasanna, H. M. Kandamby, \\ D. G. K. Madusanka, and R. A. R. C. Gopura
}

Bionics Laboratory, Department of Mechanical Engineering, University of Moratuwa, Moratuwa, Sri Lanka

Correspondence should be addressed to C. L. Semasinghe; chathural.semasinghe@gmail.com

Received 20 May 2017; Revised 21 October 2017; Accepted 16 November 2017; Published 1 March 2018

Academic Editor: Yangmin Li

Copyright (c) 2018 C. L. Semasinghe et al. This is an open access article distributed under the Creative Commons Attribution License, which permits unrestricted use, distribution, and reproduction in any medium, provided the original work is properly cited.

\begin{abstract}
This paper proposes a multi-DoF hybrid-powered transradial robotic prosthesis, named HyPro. The HyPro consists of two prosthetic units: hand and wrist that can achieve five grasping patterns such as power grasp, tip grasp, lateral grasp, hook grasp, and index point. It is an underactuated device with 15 degrees of freedom. A hybrid powering concept is proposed and implemented on hand unit of HyPro where the key focus is on restoration of grasp functions of biological hand. A novel underactuated mechanism is introduced to achieve the required hand preshaping for a given grasping pattern using electric power in the pregrasp stage and body power is used in grasp stage to execute the final grasping action with the selected fingers. Unlike existing hybrid prostheses where each of the joints is separately controlled by either electric or body power, the proposed prosthesis is capable of delivering grasping power in combination. The wrist unit of HyPro is designed and developed to achieve flexion-extension and supination-pronation using electric power. Experiments were carried out to evaluate the functionality and performance of the proposed hybrid-powered robotic prosthesis. The results verified the potential of HyPro to perform intended grasping patterns effectively and efficiently.
\end{abstract}

\section{Introduction}

Transradial amputations refer to loss of both biological hand and wrist and are the most common form of upper extremity amputation according to statistics [1]. Traumatic accidents, vascular complications, and congenital deformity are notable causes leading to transradial amputation [1]. Transradial prosthesis is used as a wearable device by the transradial amputees. It consists of an artificial wrist and a terminal device which are either mechanically or electromechanically operated $[2,3]$. Although there had been a marked improvement in functionality, performance, and appearance of subsequent generations of transradial prostheses, the robotic counterparts are still lacking in certain areas to restore or mimic the missing segments of the biological limb [3]. In particular, most transradial prostheses are designed to perform a few of the grasping tasks with limited degrees of freedom (DoF), in comparison to the biological wrist and hand that have 27 DoF [4].

In general, effectiveness of transradial prostheses has been determined based on power consumption, weight, mobility, appearance, and size of the device [5]. These also serve as benchmarks to compare and contrast different prosthetic developments. According to literature, three main categories of transradial prostheses can be identified based on method of powering: nonpowered, body-powered, and externally powered prostheses [3]. Nonpowered or cosmetic transradial prostheses are made considering anthropometry and appearance of hand and characteristically do not have any or limited functional capabilities [6]. At present, body-powered transradial prostheses are the most popular prostheses since they are lightweight, simple in operation, easily maintained, economical, and importantly capable of restoring certain functions of the biological hand [6]. Since they can only perform maximum two grasping actions from a single terminal device, different terminal devices are required to fulfill various activities of daily living (ADL) [7]. The externally powered transradial prostheses are mostly operated by means of electric and pneumatic actuators. These prostheses exhibit higher functional capabilities compared to other two categories. However, externally powered transradial prostheses are typically limited in operation due to 
high power consumption, complexity in operation, reliability, bulkiness, and overweight of the device [7].

Elimination of these limitations may enable the researchers and developers to derive a suitable replacement for a missing limb segment. One approach in realizing this is to introduce a hybrid powering concept where the device is given the capability of functioning with both body and external power [8]. There are no considerable applications of this concept in context of prosthetic limbs, and yet hybrid-powered prostheses are in the introductory phase of its life cycle. In literature, handful number of researches are identified in relation to hybrid-powered prosthesis where most of them use different units in combination that operate independently by either electric or body power $[9,10]$.

Therefore, this paper proposes a novel hybrid powering concept with the key focus on grasp restoration by actuating prosthetic thumb and fingers using both electric and body power. In addition, a novel underactuated mechanism is proposed for the prosthetic hand unit to perform different grasping patterns. The notion of adaptive grasping is also considered for the development of prosthetic fingers. The mechanism uses electric power to achieve hand preshaping according to the intended grasping pattern and body power to execute the desired grasping action. Here, the robotic prosthesis is designed to carry out five grasping patterns, namely, power grasp, tip grasp, lateral grasp, hook grasp, and index point which are commonly used in ADL. As a result of introducing the hybrid powering concept in the form of a novel finger actuation mechanism, the prosthetic hand only requires two electric actuators to perform the selected grasping patterns. Here one electric motor is used to flex and extend the three-digit prosthetic fingers while the second electric motor is used to arrange thumb position. Therefore, the proposed prosthetic hand unit is essentially an underactuated device with $15 \mathrm{DoF}$. The shoulder retractionprotraction is used to deliver the required body power to complete the grasping action. The prosthetic wrist unit proposed in this paper is fully electric-powered unit that has only two DoF controlled by two servomotors. The fully assembled prosthesis is designed to be worn by a transradial amputee using a socket placed on the residual limb and a commonly available harness mechanism is used to transmit the body power.

The paper is organized as follows. Section 2 reviews the state-of-the-art transradial prostheses to identify their capabilities and shortcomings. Section 3 briefly discusses motion requirements for transradial amputee with anatomical and biomechanical factors. Section 4 presents the design of the proposed transradial prosthesis. The mechanism of the HyPro is described in Section 5 and the experiments are presented in Section 6. Section 7 discusses the experimental results. Section 8 concludes the paper.

\section{Related Work}

One of the key limiting factors of transradial robotic prosthesis is its weight. It can be found from the literature that there is a direct relationship between device weight and number of active DoF [8]. As an example, devices such as
DEKA RC Gen3 arm [18], DLR/HIT II hand [19, 20], and MANUS hand [21] show higher weights than the devices like uGrip II [22], i-limb ultra [23], and Michelangelo [24] because of the availability of higher active DoF. Generally, this occurs due to the consequences of increasing number of actuators to achieve high active DoF. In response, the researchers have come with underactuated mechanism which couples the multiple movements to single actuator. However, such advancements have also failed to achieve significant weight reduction due to added weight of additional mechanical coupling and/or linkage systems [8]. Generally, the selection of required number of actuators depends on the desirable grasping patterns for which the robotic prosthesis is designed. It is important to have independently controlled actuators in developing a dexterous hand with individual finger movements. However, it is possible to achieve an array of grasping pattern actuation by using the coupling strategies such as differential clutching and linkage mechanism which minimizes the actuators required to operate the prosthesis.

Majority of prostheses are developed based on tendonbased and linkage-based mechanisms to achieve finger movements [8]. Ability to miniaturize the end effector is the key advantage of the tendon-based mechanisms used in the most transradial prosthetic devices. This advantage is mainly due to the ability of placing the actuators away from joints of fingers. In addition, the tendon-based mechanism helps in reducing bulkiness of the end effector while reducing the complexity of operation of prosthetic fingers [8]. However, this mechanism is known to have some drawbacks as well. The friction between tendon and tendon enclosures or sliding groves results in inefficiencies in power transmission. The durability of tendon is also a major concern. The four-bar linkage is the most common linkage-based mechanism found in the transradial prostheses. Such mechanisms generally showcase efficient power transmission while producing high grasping force and good grasp stability. However, prosthetic fingers or the end effectors are bulky when considering most prosthetic hands. In addition, the complexity of operation and higher weight are other factors associated with linkagebased mechanism. However, new linkage-based concepts have now been developed for underactuated mechanisms where some devices enable the passive finger control and enhance the object grasping capabilities. As an example, AR hand III is a novel anthropomorphic robot hand which has 15 joints driven only by three motors which is purely based on underactuated mechanism [25].

When powering of the robotic prostheses is considered, body-powered prostheses like HOSMER HOOK model $88 \mathrm{X}$ and OTTOBOCK hooks are light weight and simple to operate $[24,26]$. However, they are limited to restoring a few grasping patterns mainly due to the usage of body power to operate. The electrically powered prostheses like i-limb ultra, Bebionic 3, and Michelangelo have more functional capabilities than body-powered device but have limitations arising due to bulkiness, weight, and complexity of operation $[23,24,27]$. The major drawback of those devices is their reliance on battery power. By combining favorable features found in each prosthetic limb into one prosthesis, most of the limitations can be mitigated. 


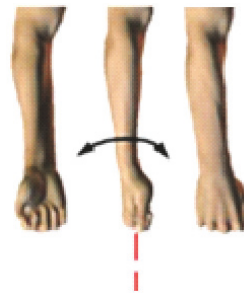

(a)

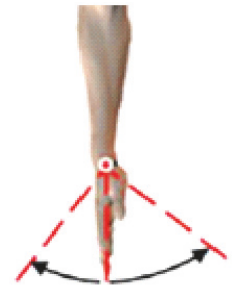

(b)

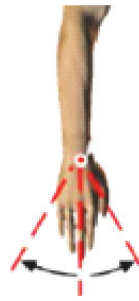

(c)

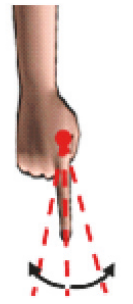

(d)

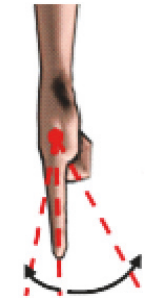

(e)

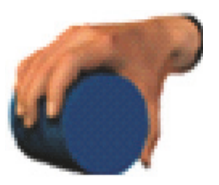

(f)

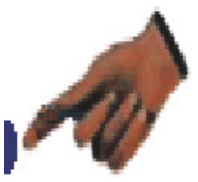

(g)

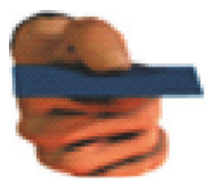

(h)

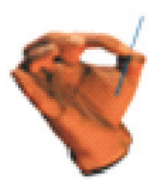

(i)

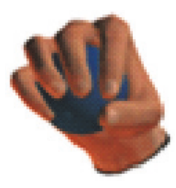

(j)

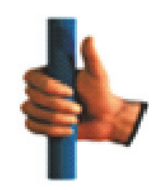

(k)

FIGURE 1: Motions of below elbow extremity (adapted from [14]) and common grasping patterns (adapted from [15]). (a) Forearm supinationpronation, (b) flexion-extension of hand, (c) radial-ulnar deviation of hand, (d) finger abduction-adduction, (e) finger flexion-extension, (f) power grasp, (g) index point, (h) lateral grasp, (i) tip grasp, (j) chuck or spherical grasp, and (k) Hook grasp.

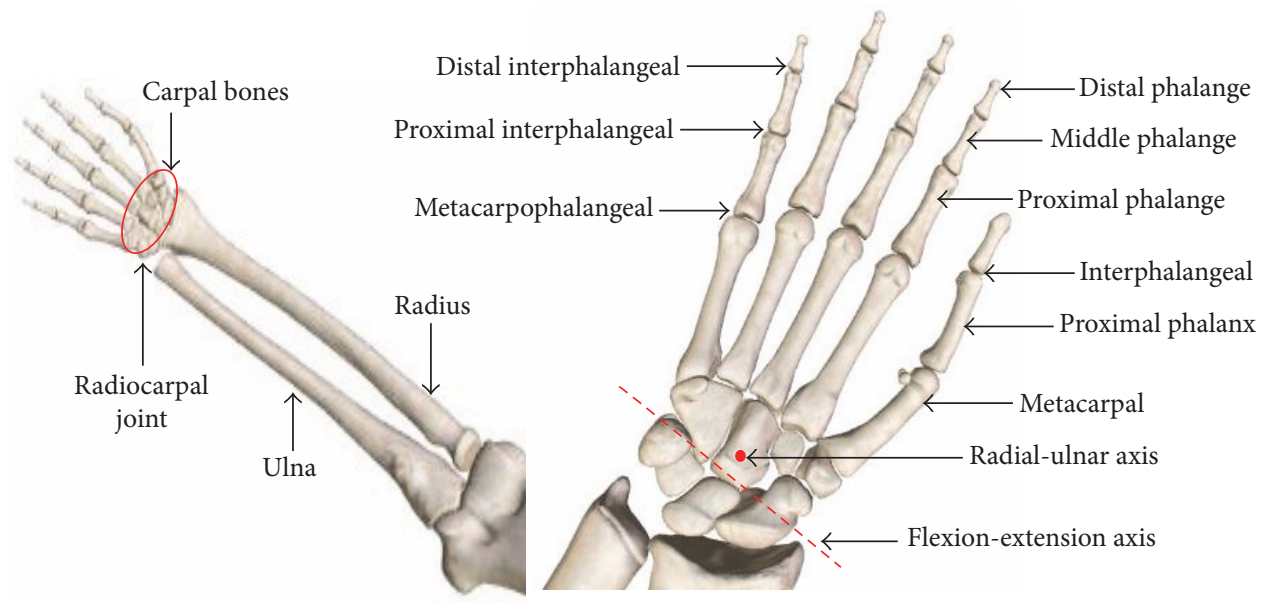

Figure 2: Anatomy of wrist and hand.

\section{Motion Requirement of Transradial Amputee}

A transradial prosthesis should ideally restore the motion requirement of the biological hand and wrist. In order to achieve this task, anatomy and biomechanics of the upper limb should be closely studied. The following section summarizes the salient aspects considered in developing the proposed hybrid-powered transradial prosthesis.

The below elbow upper limb consists of forearm, wrist, and hand. The radioulnar joint in the forearm facilitates wrist supination-pronation (see Figure 1). The wrist consists of two joints, namely, radiocarpal and midcarpal joints, which are located at the distal ends of the radial and ulna bones (see Figure 2). Radiocarpal joint allows flexion-extension and radial-ulnar deviation at the wrist [28]. The range of motion (RoM) for each DoF at wrist joint discussed above is summarized in Table 1.
Human hand consists of 27 bones, 29 joints, more than 30 muscles, and tendons to actuate the joints for the purpose of motion generation [29]. In order to handle various objects, joints of fingers are arranged in different configurations. All 3-digit fingers are comprised of metacarpophalangeal (MP), proximal interphalangeal (PIP), and distal interphalangeal (DIP) joints while thumb has only interphalangeal (IP) and metacarpophalangeal (MP) joints [12] as shown in Figure 2.

The joints in the five fingers provide $21 \mathrm{DoF}$ for grasping objects that may vary in size, shape, and weight. The thumb has five DoF whereas other fingers only four. The flexionextension of fingers are generated by three separate joints [12]. The abduction-adduction of fingers is only generated at the MCP joint. Table 2 lists RoM of each finger with respect to rest position as indicated in Figures 1(d) and 1(e).

The hand is capable of generating required articulation to support ADL. There are numerous finger arrangements, known as grasping patterns to perform several grasping 


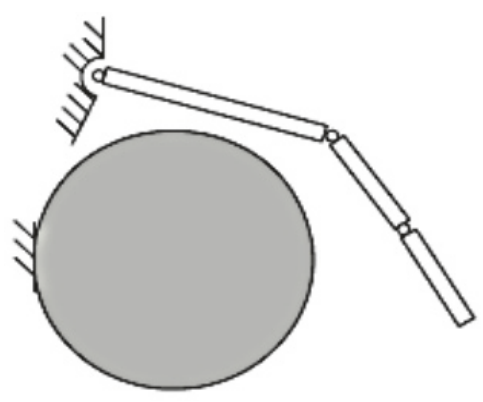

(a)

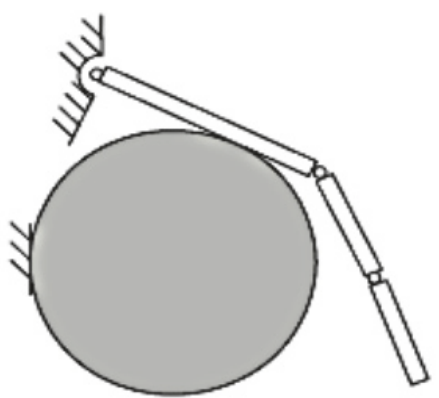

(b)

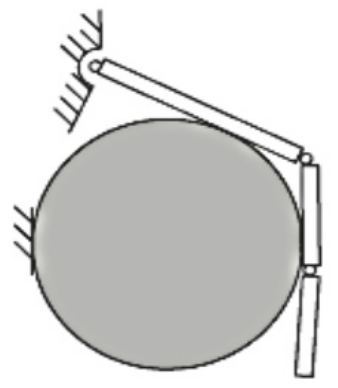

(c)

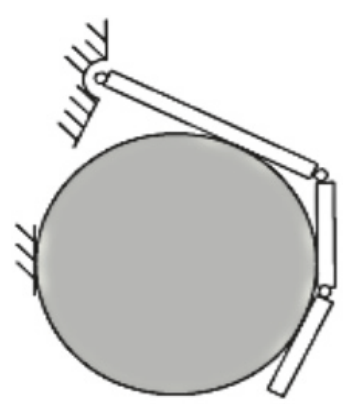

(d)

FIGURE 3: Sequence of steps followed to grasp a cylindrical object [16]: (a) approach of the finger, (b) contact of the first phalange, (c) contact of the second phalange, and (d) contact of the third phalange.

TABLE 1: Range of motions of wrist $[11,12]$.

\begin{tabular}{lc}
\hline Motion & Range \\
\hline Flexion-extension & $0^{\circ}-75^{\circ} / 0^{\circ}-\left(-70^{\circ}\right)$ \\
Radial deviation-ulnar deviation & $0^{\circ}-20^{\circ} / 0^{\circ}-\left(-35^{\circ}\right)$ \\
Supination-pronation & $0^{\circ}-90^{\circ} / 0^{\circ}-\left(-90^{\circ}\right)$ \\
\hline
\end{tabular}

actions. The main function of the prosthesis is to mimic the most important grasping patterns used in day-to-day life [30]. During the grasp action, the fingers undergo two main stages which are pregrasp and grasp execution [31]. In the pregrasp stage, the fingers arrange for achieving the desired grasping pattern while reorienting the hand. The action of gripping the target will be performed during the grasp execution stage [31]. For example, when an average person wants to hold an iron, first the hand gets prepared for grasping during its pregrasp stage which is in the reachto-grasp motion. Then grasp is executed by holding the iron by the desired grasp posture which is power grasp [31]. In the ADL, power grasp, index point, and lateral grasp are frequently used grasping patterns which are shown in Figures $1(\mathrm{f})-1(\mathrm{~h})[30]$. According to the literature, power grasp is the mostly employed grasping pattern with a frequency of $40 \%$ of $\operatorname{ADL}[30,32]$. Furthermore, frequencies of use of index finger extension, precision grasp, lateral grasp, and hook grasp are $13 \%, 12 \%, 7 \%$, and $0.2 \%$, respectively [15].

Finger adaptation is considered to be the most important feature when developing a prosthesis. Most of the prosthetic fingers are developed to generate human-like grasping action. During grasping of a cylindrical object, the phalanges of the prosthetic or biological finger follow the basic sequence of operations shown in Figure 3 [16, 33].

\section{Design of HyPro}

The HyPro consists of two major units, referred to as prosthetic wrist unit and prosthetic hand unit. Figure 4 illustrates the complete CAD model of the proposed hybrid powering transradial prosthesis without the socket.

The wrist unit is designed to operate using external power while the prosthetic hand uses a combination of both electric and body power. The hybrid actuation and underactuated

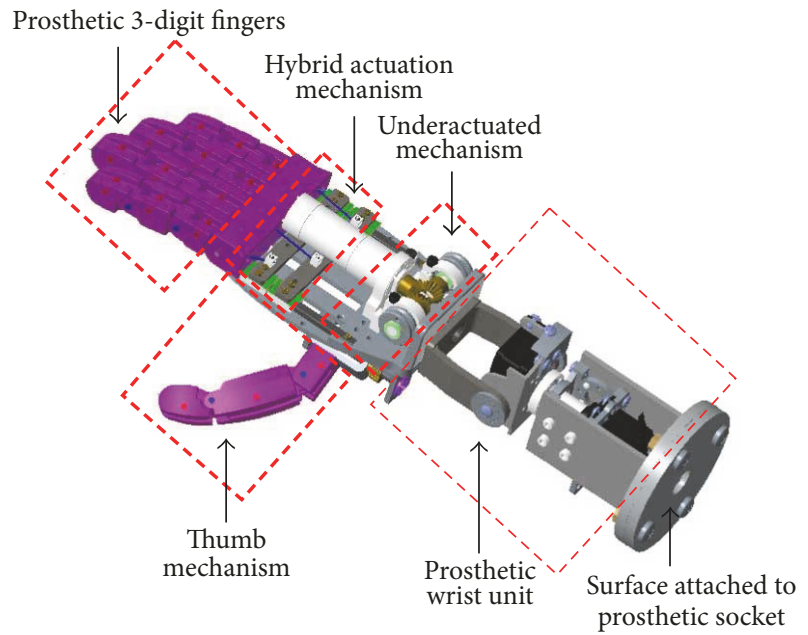

Figure 4: CAD model of HyPro.

mechanisms are located on the dorsal side of the prosthetic hand unit. The electric power is used for hand preshaping to perform the desired grasping pattern while body power is used to execute the grasp for picking objects. The grasping modes and respective powering methods used by HyPro are tabulated in Table 3.

The design details of HyPro are presented under five subsections: prosthetic wrist design, prosthetic 3-digit finger design, prosthetic thumb design, design of hybrid actuation mechanism, and design of underactuated finger flexionextension mechanism.

4.1. Prosthetic Wrist Design. The proposed design for the prosthetic wrist includes two active DoF, namely, supinationpronation and flexion-extension. The main structure of the wrist can be simplified to a mechanism consisting of two motors which work as two separate units. As shown in Figure 5 supination-pronation unit and flexion-extension unit are actuated using two servomotors, servomotor 1 (HS$5685 \mathrm{MH}$, Hitec servomotor) and servomotor 2 (HS-5585MH, Hitec servo motor), respectively. Each unit is designed to perform separate motions that can mimic the biological counterpart. The servomotor 1 is held by a servoblock attached to an aluminium structure which has been consolidated on 


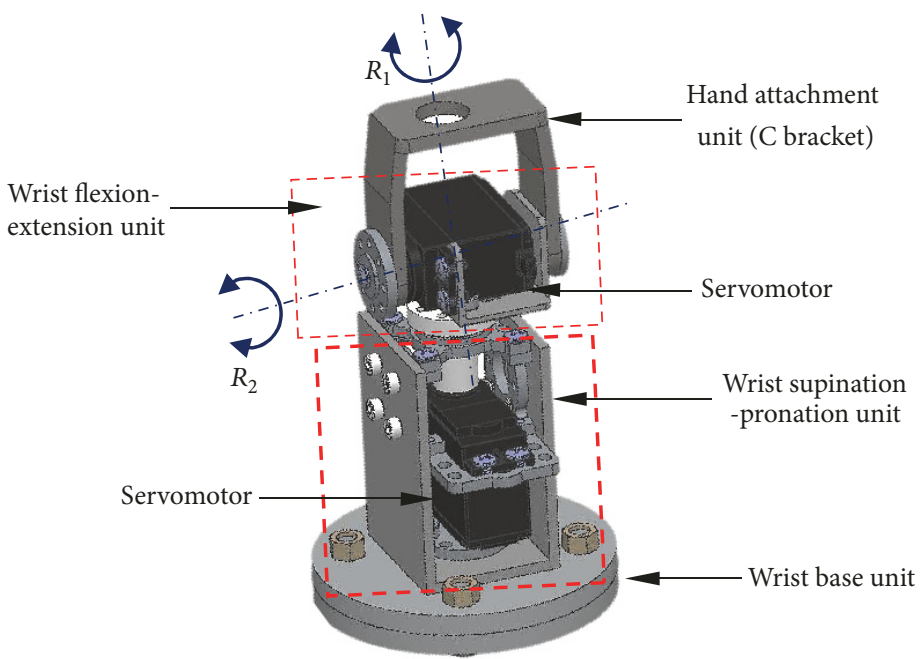

FIGURE 5: 2-DoF prosthetic wrist of HyPro.

TABLE 2: RoM of fingers $[11,13]$.

\begin{tabular}{|c|c|c|c|c|c|c|}
\hline \multirow{2}{*}{ Motion/joint } & & \multicolumn{5}{|c|}{ Finger } \\
\hline & & Thumb & Index & Middle & Ring & Little \\
\hline \multirow{3}{*}{ Flexion-extension } & MCP & $0^{\circ}-70^{\circ}$ & $\left(-30^{\circ}\right)-90^{\circ}$ & $\left(-20^{\circ}\right)-90^{\circ}$ & $\left(-30^{\circ}\right)-90^{\circ}$ & $\left(-30^{\circ}\right)-90^{\circ}$ \\
\hline & PIP & - & $0^{\circ}-120^{\circ}$ & $0^{\circ}-120^{\circ}$ & $0^{\circ}-120^{\circ}$ & $0^{\circ}-120^{\circ}$ \\
\hline & DIP & $0^{\circ}-90^{\circ}$ & $0^{\circ}-80^{\circ}$ & $0^{\circ}-80^{\circ}$ & $0^{\circ}-80^{\circ}$ & $0^{\circ}-80^{\circ}$ \\
\hline Abduction-adduction & MCP & $\left(-50^{\circ}\right)-40^{\circ}$ & $\left(-25^{\circ}\right)-20^{\circ}$ & $\left(-25^{\circ}\right)-20^{\circ}$ & $\left(-25^{\circ}\right)-20^{\circ}$ & $\left(-25^{\circ}\right)-20^{\circ}$ \\
\hline Opposition - reposition & $\mathrm{CMC}$ & $\left(-9^{\circ}\right)-31^{\circ}$ & - & - & - & - \\
\hline
\end{tabular}

TABLE 3: Grasping and powering methods of HyPro.

\begin{tabular}{lc}
\hline Grasping mode & Powering method \\
\hline Power grasp & Body power \\
Index push & Electric power \\
Tip grasp & Body and electric power \\
Hook grasp & Electric power \\
Lateral grasp & Body power \\
\hline
\end{tabular}

the wrist base unit. The servoblock contains an aluminium hub to transmit torque from the motor to prosthetic hand through the flexion-extension unit (see Figure 5). Flexionextension unit is directly coupled with the hub of servoblock to transmit the torque of servomotor 1 . The servomotor 2 is used to generate torque for wrist flexion-extension motion and the C-bracket is used to attach the prosthetic hand to the flexion-extension unit.

4.2. Prosthetic 3-Digit Finger Design. The fingers are designed considering 95th percentile anthropometric data of male population in the world [34]. The prosthetic finger closely matches the biological human finger from a functional point of view. The passive extension and adaptive grasping of phalanges have been achieved by employing elastic straps on the dorsal side of the prosthetic fingers. Different strap patterns were introduced between phalanges and the overall stiffness of the elastic straps was found to be $38.8 \mathrm{~N} / \mathrm{m}$ from the physical experiments conducted on prosthetic finger samples. All the prosthetic fingers in HyPro are designed to work using a tendon-based mechanism where the finger gets flexed when the flexor tendon is pulled. Therefore, the prosthetic hand is voluntarily opened because of the extended fingers due to relaxation of elastic straps under zero force on the flexor tendons. Figure 6 shows the 3D model of the proposed prosthetic finger. Each of the segments of the prosthetic finger was fabricated from PLA material using 3D printing technology and $3 \mathrm{~mm}$ stainless steel pins were inserted to form the hinges. Finite element simulations were performed to assess the load bearing capacity of the prosthetic finger. Stress field plots indicate that regions nearby the hinges are most vulnerable for failure. However, each of the fingers is designed to bear $5 \mathrm{~kg}$ of force with a safety factor of 2 .

4.3. Prosthetic Thumb Design. As in the case of 3-digit fingers, the prosthetic thumb is equipped with a tendonbased mechanism to flex the thumb. It is comprised of two phalanges similar to the biological thumb. In order to achieve passive extension and adaptive grasping abilities, two springs with different force constants were attached to each of the phalanges. The flexion-extension motion of the prosthetic thumb is achieved by pulling the flexor tendon using body 


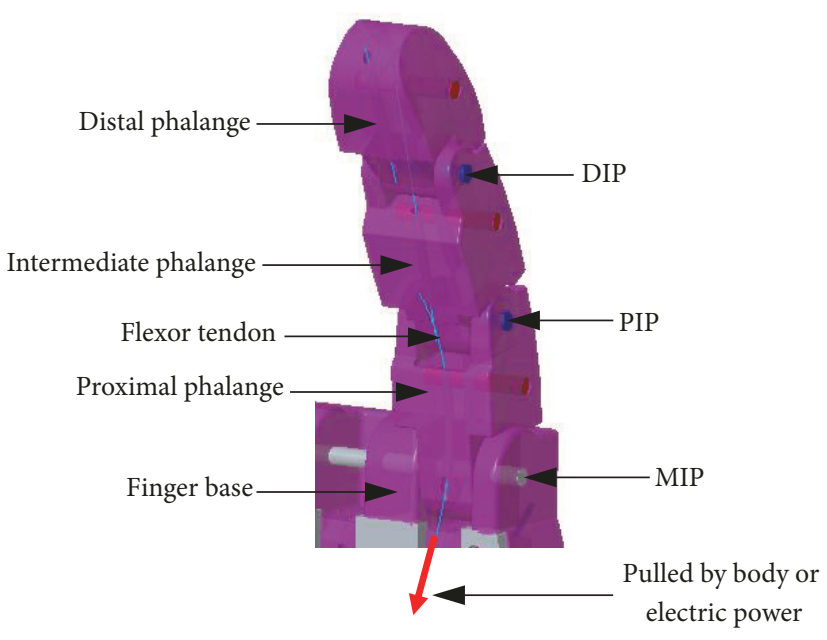

(a)

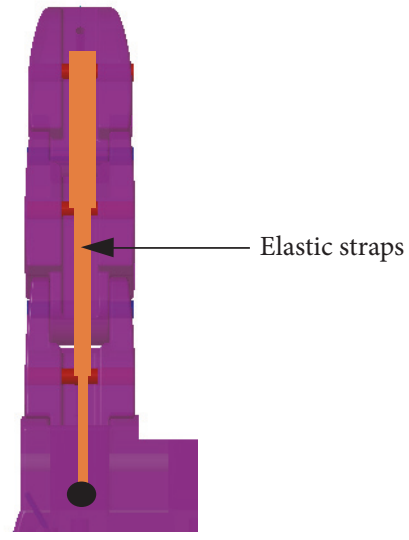

(b)

Figure 6: Model of prosthetic 3-digit finger: (a) 3-digit finger design with internal tendon flow path; (b) elastic straps arrangement of the finger model.
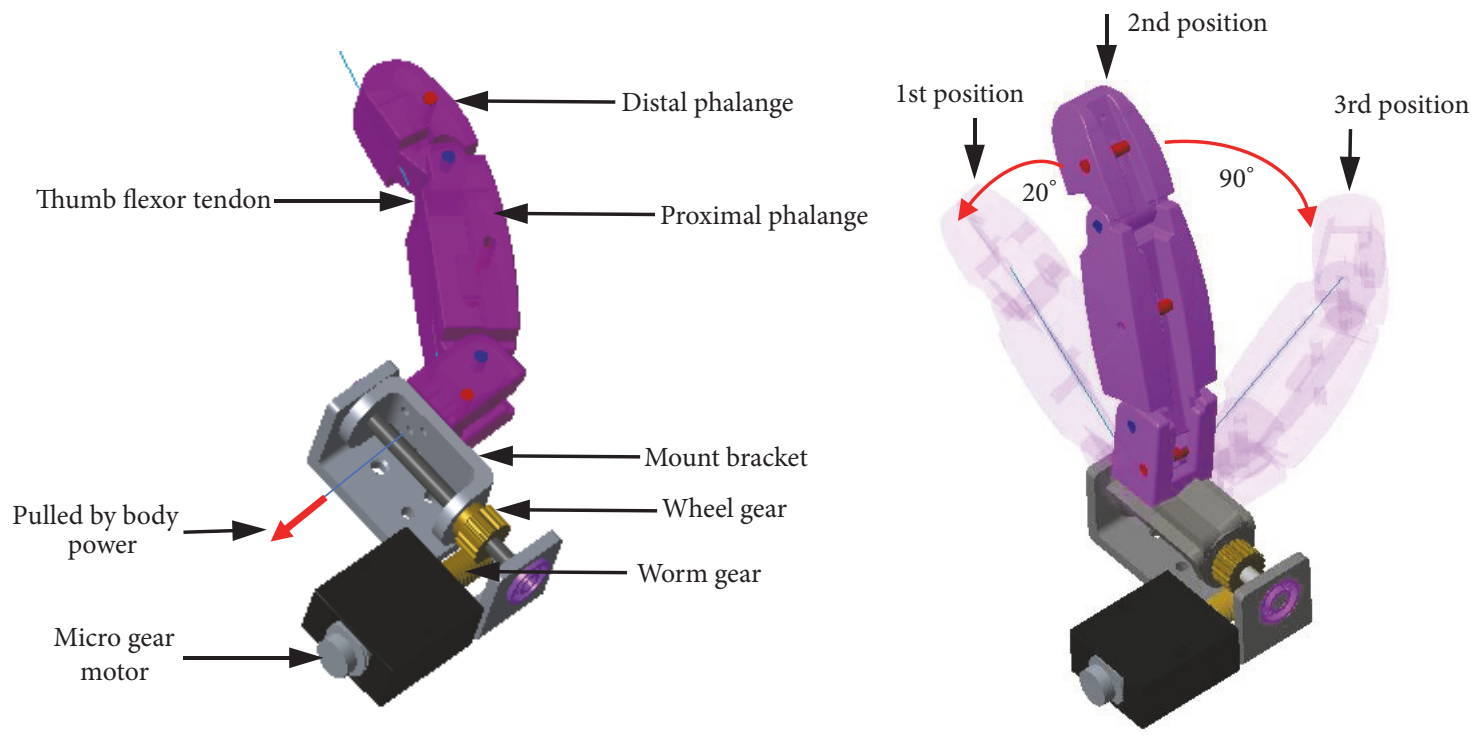

FIgURE 7: CAD model of prosthetic thumb.

power. In order to perform different grasping patterns, the thumb abduction-adduction movement is achieved using a micro gear motor (90 rpm micro gear motor with encoder). The worm and wheel gear drive provide a speed reduction of $1: 10$.

Figure 7 shows the 3D model of the proposed prosthetic thumb. HyPro is designed to perform only five key grasping patterns; hence, abduction-adduction movement is limited to three different positions to achieve desired grasping patterns. As shown in Figure 7, thumb shifts into 1st position for hand preshaping for power grasp. In order to preshape for tip grasping pattern the thumb moves to its 2 nd position. For generating the index point posture, lateral grasping and hook grasping pattern thumb will be shifted to its 3rd position.
4.4. Design of Hybrid Actuation Mechanism. The prosthetic hand uses a tendon-based mechanism to actuate all the fingers by either body power or electric power. As per the design, the prosthetic middle, ring, and little fingers are flexed and extended simultaneously while the prosthetic index and thumb operate independently. In case of the prosthetic thumb, abduction-adduction has been implemented to achieve several grasping patterns. In electric-power actuation mode, the flexion-extension of all 3-digit fingers is performed using single DC brushed motor ( $38 \mathrm{rpm}$ premium planetary gear motor with encoder). The motor rotation is used to flex only three or all four fingers as per the relevant control input. As shown in Figure 8, flexor tendon connected to each prosthetic finger is attached to the sliders, slider A and slider B. Here, slider A pulls the tendons from prosthetic middle, 


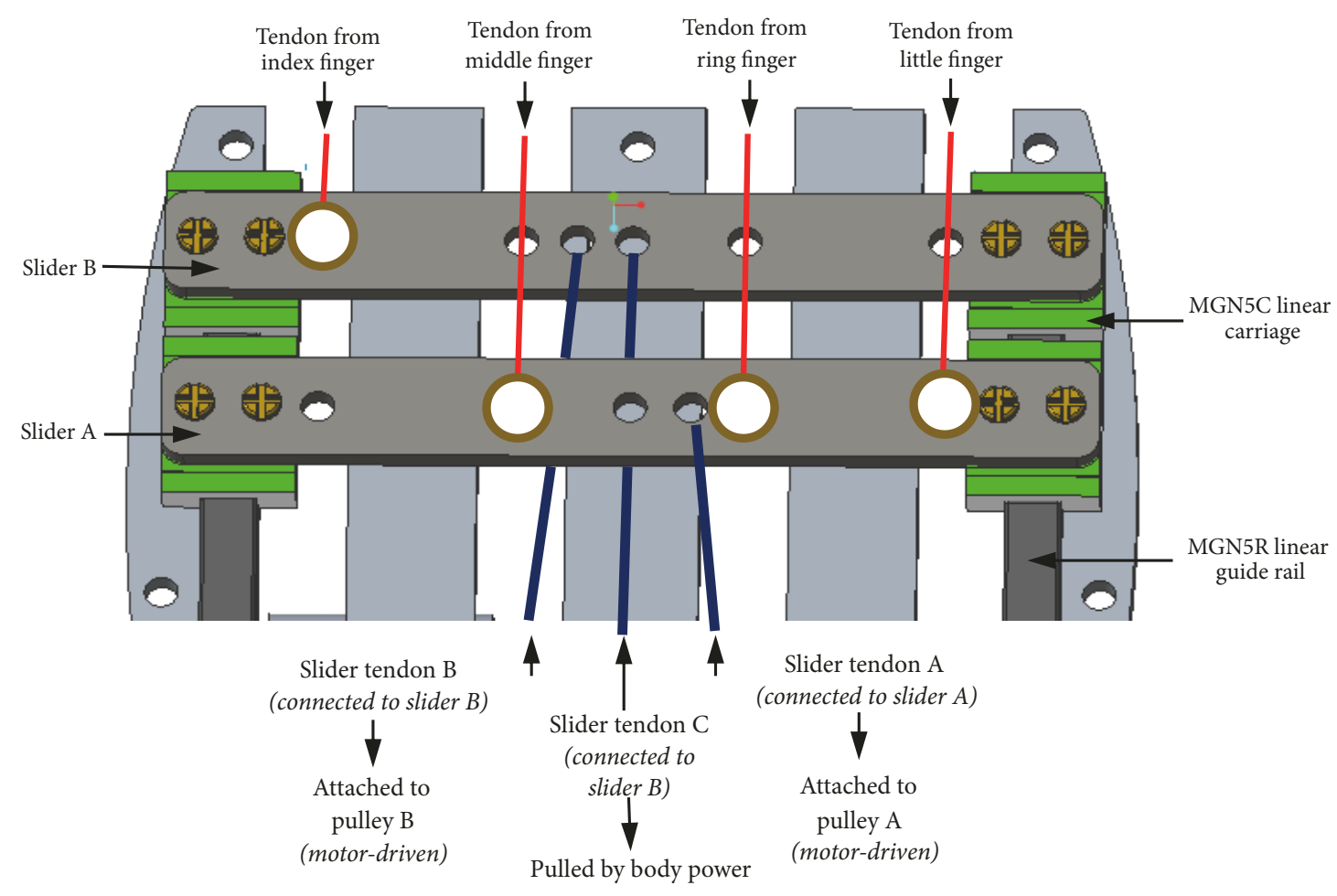

FIGURE 8: CAD model of the hybrid actuation mechanism.

ring, and little fingers while slider B pulls the tendon from prosthetic index finger.

Each slider is fixed on two carriage blocks (HIWIN MGN5C) where those carriage blocks linearly move on two linear guide rails (HIWIN MGN5R). Here both sliders can slide along the guide rail independently. Figure 8 illustrates the tendon connection to each of the sliders in HyPro. In order to flex prosthetic middle, ring and little fingers slider $\mathrm{A}$ has to be pulled by the slider tendon $\mathrm{A}$. When the slider tendon $B$ is pulled, the slider $B$ moves linearly along the linear guide rails while pushing the slider $\mathrm{A}$ as well. Therefore, pulling the slider tendon $\mathrm{B}$ causes all four fingers to be flexed. Both slider tendon A and B are pulled using the same DC brushed motor which is electrically powered. The slider tendon $C$ is designed to be pulled by body power which makes all four fingers flexed after pulling. Therefore, all four 3-digit fingers can be flexed by either pulling slider tendon B using DC brushed motor or pulling slider tendon $C$ using body power. If the slider $\mathrm{A}$ is pulled by slider tendon A using DC brushed motor, the slider tendon $\mathrm{C}$ allows only index finger to flex or extend by pulling or releasing, respectively. When the effort on pulling slider tendons is released the fingers extend due to passive extension mechanism implemented on each finger. Here, the linear carriages and linear guide rails have been selected to satisfy the space requirement and to withstand the contact forces that occur on each slider due to unsymmetrical force distribution.

4.5. Underactuated Finger Flexion-Extension Mechanism. In HyPro, an underactuated mechanism is implemented to achieve finger flexion-extension with electric power. The proposed underactuated mechanism has resulted in reduction of weight and simplification of overall control of the prosthesis. This is mainly due to elimination of motors. However, the mechanism found inside HyPro is capable of performing five different grasping patterns using a single motor with the assistance of body power. When the DC motor gets actuated either slider tendon $\mathrm{A}$ or $\mathrm{B}$ winds on the pulleys causing the fingers to flex as planned. In order to pull one slider at a given instance, a simple mechanism has been implemented using couple of bearings. As shown in Figure 9(a), combinations of one-way and radial bearings are employed to achieve independent pulley rotation.

A one-way bearing allows a shaft inside to rotate freely only in one direction. If this one-way bearing is mounted inside a radial bearing as shown in Figure 9(b), the inner racer of the radial bearing will rotate when the shaft rotates in opposite direction due to the constrain of the shaft inside the one-way bearing. Figure 10 shows the working principal of the bilateral pulley-bearing assembly which explains the independent pulley movement capability using a single DC motor. Note that the tendon attachment pulley is mounted on the outer surface of one-way bearing and the pulley will only rotate when the one-way bearing is rotating inside the radial bearing.

When the motor shaft rotates in anticlockwise direction only, pulley A will rotate while pulling the slider A. It results in flexing prosthetic middle, ring, and little fingers. If the motor rotates in clockwise direction, only pulley B will rotate. The motion causes slider B to be pulled and slider tendon A tries to unwind from the pulley to release the strain energy stored in elastic straps. However, when slider B is pulled, 


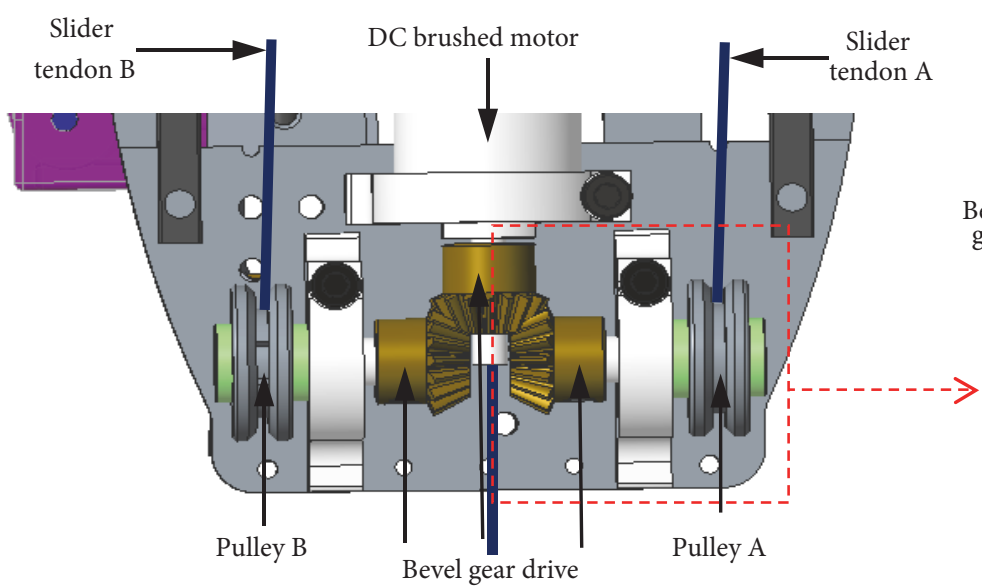

(a)

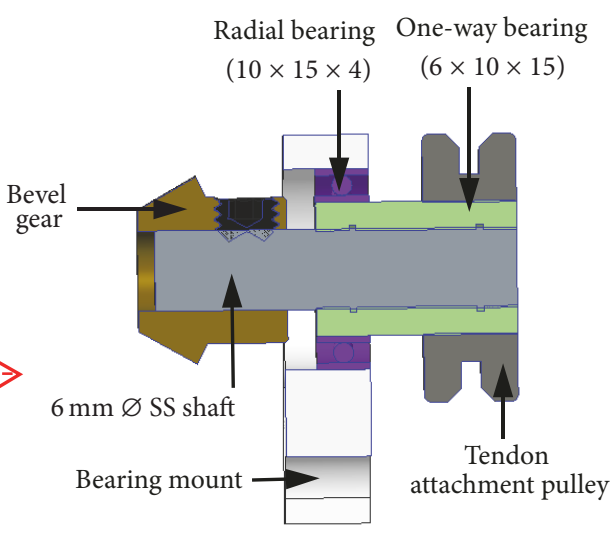

(b)

FIGURE 9: CAD model of the underactuated flexion-extension mechanism of finger.

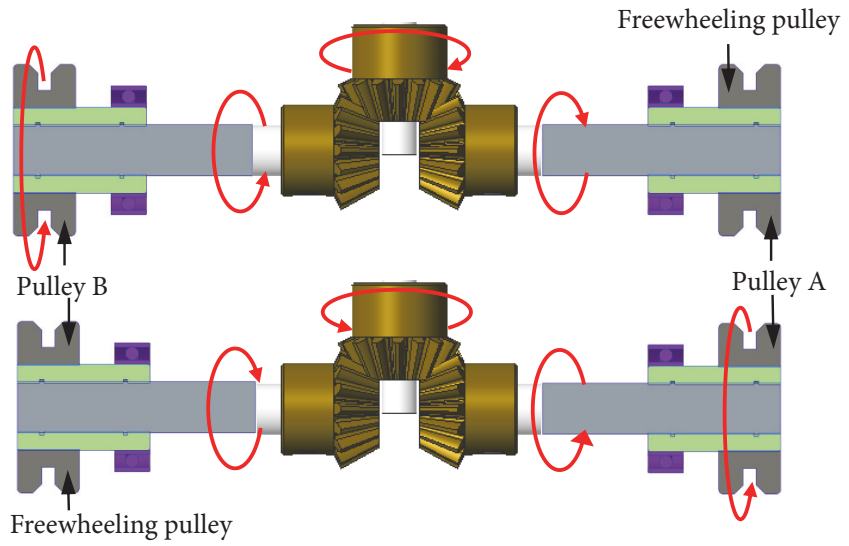

FIGURE 10: Bilateral pulley-bearing assembly of the underactuated mechanism.

slider A will also get pushed due to impending contact forces between the two sliders. As a consequence, all four fingers flex in the prosthetic hand. Then, when the shaft rotates in anticlockwise direction, the slider tendon $B$ unwinds from the pulley while slider tendon $A$ is winding on tendon attachment pulley.

4.6. Fabricated Model of HyPro. The fabricated model of the HyPro, including prosthetic hand unit, prosthetic wrist unit, prosthetic socket, and harness, is shown in Figure 11. Mass of the overall system including the battery pack (Li-Po $2200 \mathrm{mAh}$ ) is $1.8 \mathrm{~kg}$ in total. Fabricated model of HyPro hand has the $15 \mathrm{DoF}$ as discussed in the design while having the two DoF in the HyPro wrist. Table 4 shows the achieved DoF in HyPro using body power from shoulder movement and electric power with two DC motors and two servomotors.

\section{HyPro Hand Actuation Method}

In order to grasp objects, five elements of the prosthesis are to be controlled. Those include slider tendon A, slider

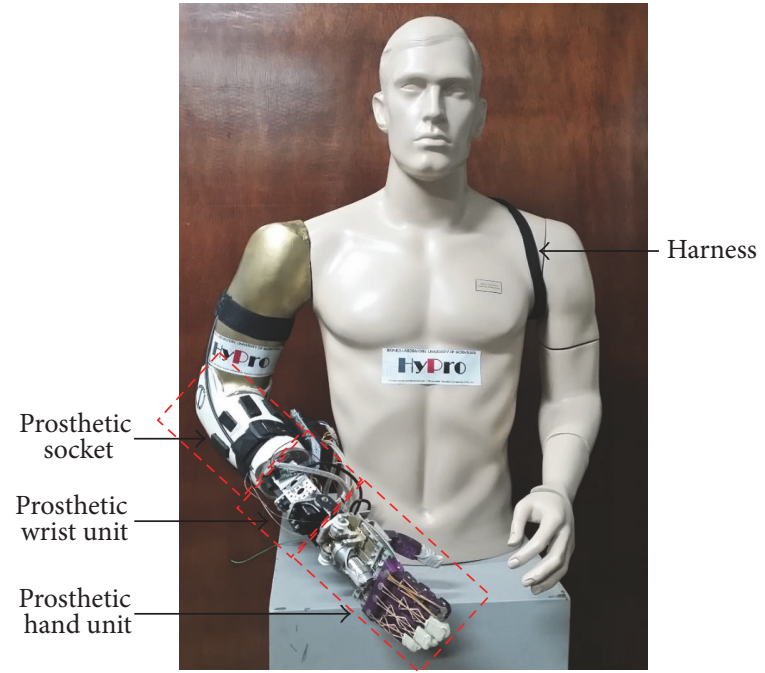

Figure 11: The HyPro.

tendon $\mathrm{B}$, thumb motor, slider tendon $\mathrm{C}$, and thumb flexor tendon. Slider tendon $\mathrm{A}$, slider $\mathrm{B}$, and thumb motor are actuated by electric power while both slider tendon $\mathrm{C}$ and thumb flexor tendon are pulled simultaneously using body movement which is shoulder protraction-retraction. Each tendon has two states which are referred to as pulled and idle states. The thumb motor is used to attain three different work positions for achieving predefined grasping patterns. Table 5 describes the sequence of steps followed to achieve the required grasping pattern.

For example, to perform the lateral grasp, fingers are initially brought into hand preshaping by flexing the threedigit fingers fully and abducting the thumb to the 3 rd position using electric power. Then the object is grasped by flexing the thumb using body power. Sequence of steps followed during lateral grasp is depicted in Figure 12. Similarly, actuation methods for other grasping patterns are obtained as per the sequences described in the Figure 13. 
TABle 4: Achieved DoF by HyPro.

\begin{tabular}{|c|c|c|c|c|c|}
\hline Unit & Component & Joint & Movement & Actuation method & DoF \\
\hline \multirow{7}{*}{ Hand unit } & \multirow{3}{*}{ Thumb } & Interphalangeal & Flexion-extension & Body power & 1 \\
\hline & & \multirow{2}{*}{ Metacarpophalangeal } & Flexion-extension & Body power & 1 \\
\hline & & & Abduction-adduction & Electric power & 1 \\
\hline & \multirow{4}{*}{$\begin{array}{c}\text { Index finger } \\
\text { Middle finger Ring } \\
\text { finger } \\
\text { Little finger }\end{array}$} & Distal interphalangeal & Flexion-extension & $\begin{array}{l}\text { Body power or } \\
\text { electric power }\end{array}$ & $1 \times 4$ \\
\hline & & $\begin{array}{c}\text { Proximal } \\
\text { interphalangeal }\end{array}$ & Flexion-extension & $\begin{array}{l}\text { Body power or } \\
\text { electric power }\end{array}$ & $1 \times 4$ \\
\hline & & Metacarpophalangeal & Flexion-extension & $\begin{array}{l}\text { Body power or } \\
\text { electric power }\end{array}$ & $1 \times 4$ \\
\hline & & \multicolumn{3}{|c|}{ Total number of DoF in HyPro hand unit } & 15 \\
\hline \multirow{3}{*}{ Wrist unit } & \multirow{3}{*}{ Wrist joint } & \multirow{2}{*}{ Radiocarpal } & Flexion-extension & Electric power & 1 \\
\hline & & & Supination-pronation & Electric power & 1 \\
\hline & & \multicolumn{3}{|c|}{ Total number of DoF in HyPro wrist unit } & 2 \\
\hline
\end{tabular}

TABLE 5: Powering methods to actuate grasping patterns.

\begin{tabular}{lccccc}
\hline \multirow{2}{*}{ Control input signal } & \multicolumn{3}{c}{ Mechanism } & \multicolumn{2}{c}{ Body power for grasp execution } \\
& \multicolumn{2}{c}{ External power for hand preshaping } & Output \\
\hline Power & Slider tendon A & Slider tendon B & Thumb motor & Slider tendon C + thumb flexor tendon \\
Tip & Idle & Idle & 1st position & Pulled & Power grasp \\
Index & Pulled & Idle & 2nd position & Pulled & Tip grasp \\
Lateral & Pulled & Idle & 3rd position & Idle & Index point \\
Hook & Idle & Pulled & 3rd position & Pulled & Lateral grasp \\
\hline
\end{tabular}

The electric power is only used to preshape the hand for achieving required grasping pattern with respect to the given control input signal. It is the same as changing terminal devices in conventional body-powered upper limb prostheses that enables grasping of different objects. Similarly, HyPro uses the same principle of grasp execution found in bodypowered prostheses where shoulder retraction-protraction is used to execute the terminal device for grasping objects. Figure 14 illustrates how the body movement is used in achieving the grasping in the conventional body-powered prosthetic devices.

\section{Experiments}

Five different experiments were carried out in order to validate the design and performance of HyPro. These tests were performed to investigate the ability of the prosthesis to grasp and handle objects. In this section, experimental setups prepared for determining kinematics prosthetic fingers, adaptive behavior of prosthetic fingers, ability to perform grasping patterns, fingertip forces, and energy consumed during grasping patterns were systematically analyzed.

6.1. Experiment on Kinematics of Prosthetic Fingers. Prosthetic fingers are the key elements in any upper limb prosthesis. The fingers have to be properly designed to grasp an object with a selected grasping pattern. Therefore, the design of the prosthetic finger closely follows the biological finger to generate human-like motions. In order to validate the proposed design, a mathematical model of the prosthetic finger was prepared on a simulation platform, "Working Model 2D." Critical parameters for the model to be identified are length of digits in the prosthetic fingers and force constants of elastic straps. Model was used to evaluate the kinematics of the phalanges such as angular displacement of interphalangeal joints. In order to evaluate the actual prosthetic finger movement, an experiment was conducted using a movement analysis software package, "Kinovea." At first, markers were placed on each digit of prosthetic index finger (see Figure 15). Then, a high frame rate camera (120 fps @ 1080p) was used to record a video footage of flexion-extension of index finger. Finally, the marker movements were used to determine the interphalangeal angular displacements using Kinovea. Results are presented in detail in Section 7.

6.2. Experiment on Adaptive Behavior of Prosthetic Fingers. In order to identify the adaptive grasping behavior of a prosthetic finger, HyPro was used to grasp three cylindrical objects with different diameters. The cylinders were held firmly and the HyPro was given the input to grasp each object by gradual means. The sequence of snapshots extracted from a high frame rate video footage is included in Section 7 to showcase the adaptive grasping behavior of the proposed prosthetic fingers. 

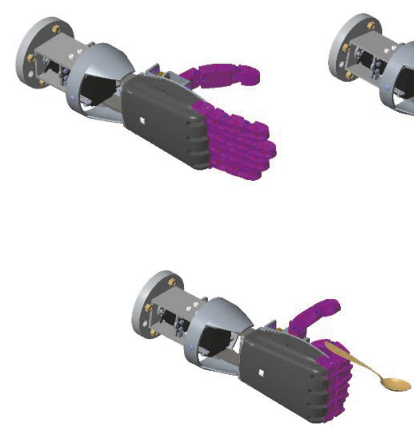
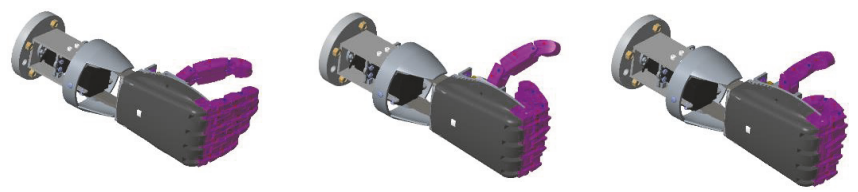

(a)
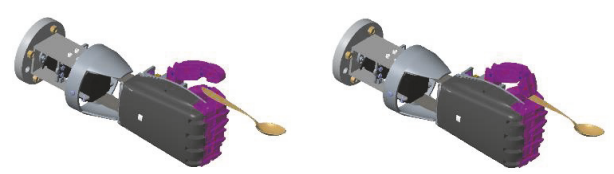

(b)

FIGURE 12: Holding a spoon using lateral grip: (a) hand preshaping by electric power; (b) grasp execution by body power.

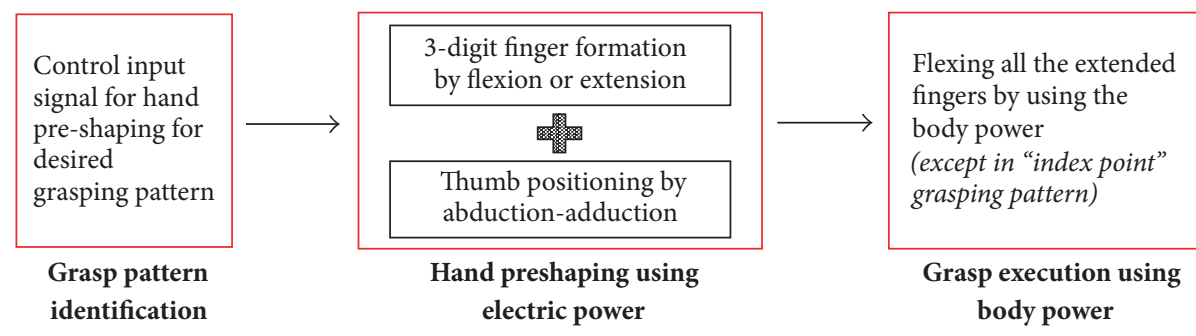

FIGURE 13: Sequence of achieving the desired grasping pattern.
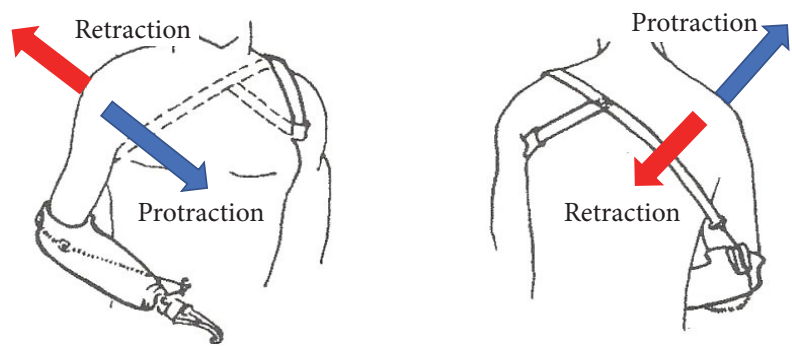

FIGURE 14: Shoulder motion to activate the prosthesis (adapted from [17]).

6.3. Experiment on Ability to Perform Grasping Patterns. The fabricated prosthesis hand was assigned with different tasks to evaluate the capability of achieving each of the five grasping patterns. In order to test the ability to perform power grasp, HyPro was given a water bottle to hold. Tip grasp, lateral grasp, and hook grasp were tested by making HyPro to hold a pen drive, a spoon, and a bag, respectively. The results of achieving those patterns are discussed in Section 7.

6.4. Experiment on Fingertip Forces. An experiment was conducted with prosthetic fingers to find the force exerted on objects during the grasping from the fingertip of prosthetic finger. During the experiment, the data on output forces was collected from all five fingers at the given instances of input forces given through the body-powered cable. The outcome of the results gathered has been briefly discussed in Section 7.

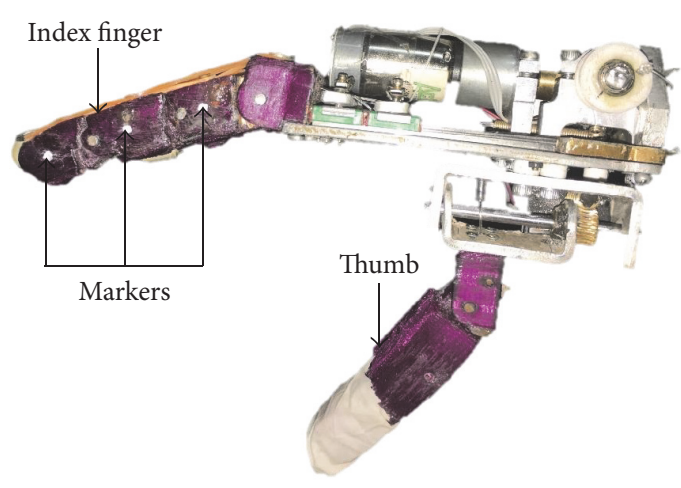

FIGURE 15: Marker placement on prosthetic index finger.

6.5. Experiment on Energy Consumed during Grasping Patterns. Energy consumption is a key measure to verify the effectiveness of HyPro over fully externally powered prostheses. The hybrid actuation mechanism is intended to increase battery life and reduce the effort exerted by body to actuate the prosthesis. In order to identify the energy requirement for hand preshaping during the pregrasp stage, an experiment setup was developed. It was subsequently used to determine the power requirement to perform all five grasping patterns. In order to measure the power consumption, current sensors were employed with a constant voltage input. A microcontroller (ATmega2560) was used to record data over a period of time.

The total energy consumption for each grasping pattern was then calculated using the current values measured for 


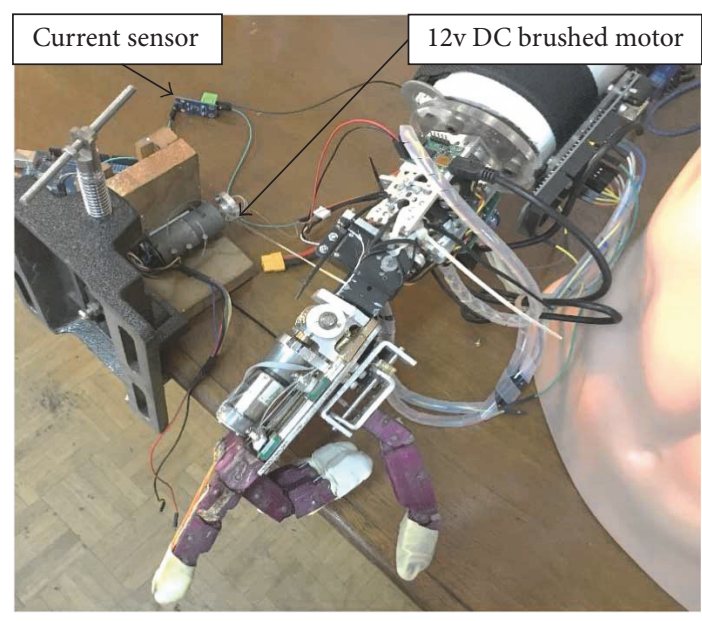

FIGURE 16: Experimental setup for obtaining current measure.

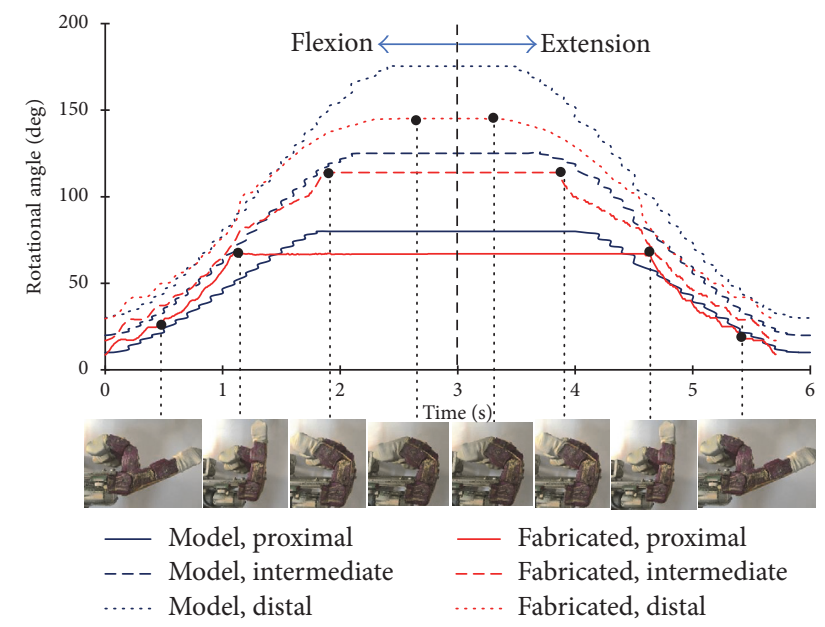

FIGURE 17: Kinematics of index finger.

a given duration. In order to find the power requirement to perform a desired grasping action, a separate $12 \mathrm{v} \mathrm{DC}$ brushed motor was used to imitate the effort exerted by body power. The tendons supposed to be pulled by the body were attached to the motor shaft. The current measures were obtained using the microcontroller while performing desired grasping actions. The experimental setup for obtaining the current measures to determine energy consumption is shown in Figure 16.

\section{Results and Discussion}

In this section, the results obtained from the five different experiments described in the previous section are briefly discussed.

7.1. Kinematics of Prosthetic Finger. The graph in Figure 17 shows the results obtained for rotational angles for both simulated model and fabricated model of index finger for the given time instances with reference to finger base. It is noted that proximal digit model stops rotating after $1.7 \mathrm{~s}$ while intermediate digit rotates until $2.1 \mathrm{~s}$ during the flexion stage. The distal digit model rotates until $2.5 \mathrm{~s}$ during the flexion stage. Thus, it shows that the proximal digit stops rotating first, then intermediate digit, and finally the distal digit during the simulated model finger flexion. Although the time values obtained on fabricated finger model are leading compared to simulated model still it has shown the same sequence of digit rotations during the flexion stage.

During the extension stage in both simulated model and fabricated finger, the distal digit starts rotating first, then intermediate digit and finally the distal digit. When the finger comes to its rest position all digits stop rotation with reference to finger base. This validates that the prosthetic finger movement follows the human finger flexion-extension from both simulated model and fabricated model. The Figure 17 also shows the sequence of snapshots of fabricated prosthetic finger flexion-extension followed by the results shown in the graph.

7.2. Adaptive Grasping by HyPro Hand. The prosthetic fingers were given 3 tasks to review the adaptive grasping of fingers. As described in the experimental setup, 3 sequences of images (see Figure 18) were obtained to show the adaptive grasp movement in prosthetic fingers.

In all the tasks when the fingers are given an object to grasp first proximal digit gets contacted with object surface and stops rotating around metacarpi-phalangeal joint while other intermediate and distal digits are rotating to get contacted with the object. Then the intermediate digit gets contacted with object surface and stop rotating around proximal interphalangeal joint. Finally, the distal digit rotates until it gets contacted with the object. Therefore, all these movement patterns during the object grasping show the adaptive grasping of objects by the HyPro hand.

7.3. Grasping Patterns and Postures Achieved by HyPro. In order to identify the capability of HyPro hand in achieving the grasping patterns several tasks were given to observe the functionality. Figure 19 shows the set of activities achieved by HyPro for given tasks which are rest position, power grasp, index point, tip grasp lateral grasp, and hook grasp. All the tasks were achieved successfully by the HyPro.

It verified that the HyPro is capable of achieving all five grasping patterns. From the statistics, the considered set of grasping patterns has frequency of $72.2 \%$ out of all grasping patterns used in ADL $[13,30]$. Hence, HyPro is capable of performing more than $70 \%$ of task found in ADL.

7.4. Force Exerted by Prosthetic Fingertip. From the results of the experiment, average fingertip force is plotted against input force given by pulling of the tendon (see Figure 20). It is found that at least $3.34 \mathrm{~N}$ force is needed for starting the flexion movement in prosthetic fingers and prosthetic finger exerts $0.1 \mathrm{~N}$ force on the object during the grasp with the increment of $1 \mathrm{~N}$ force of body power. In order to reduce the frictional losses, an efficient design of tendon flow paths is needed in future developments. 

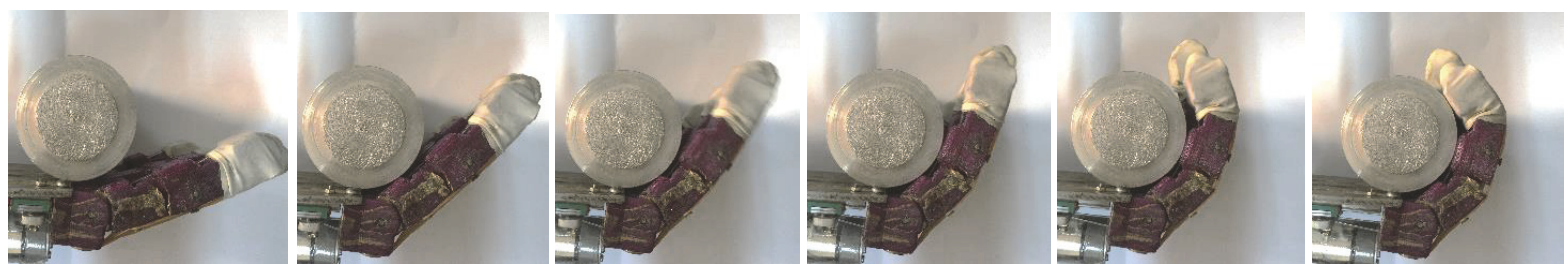

(a)
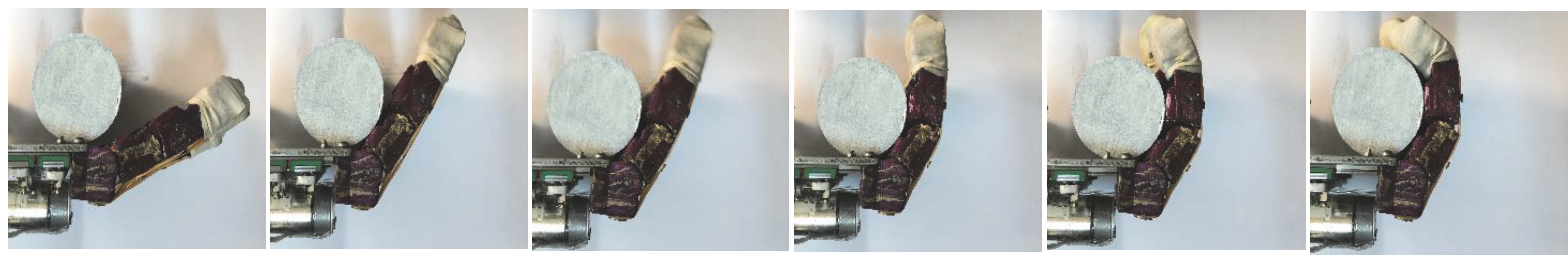

(b)
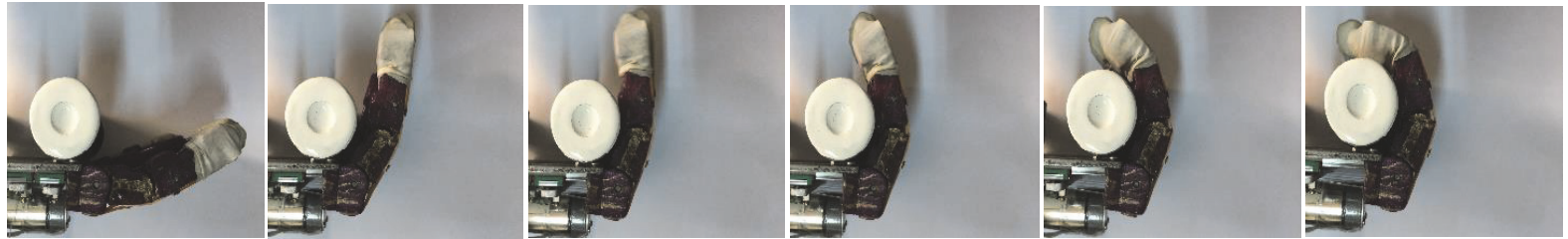

(c)

FIGURE 18: Adaptive grasping of objects: (a) grasping $52 \mathrm{~mm}$ diameter shaft; (b) grasping $45 \mathrm{~mm}$ diameter shaft; (c) grasping $32 \mathrm{~mm}$ diameter shaft.

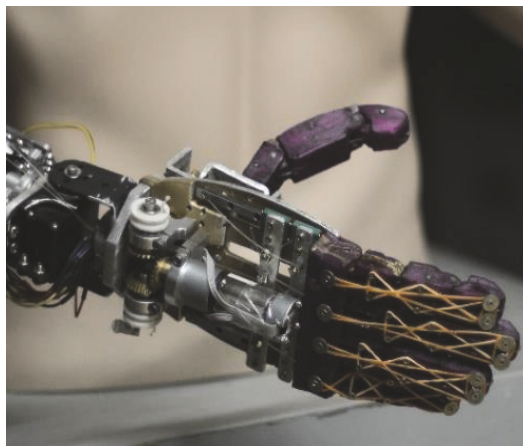

(a)

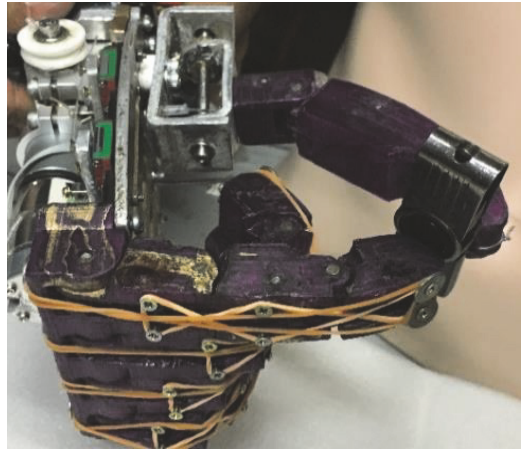

(d)

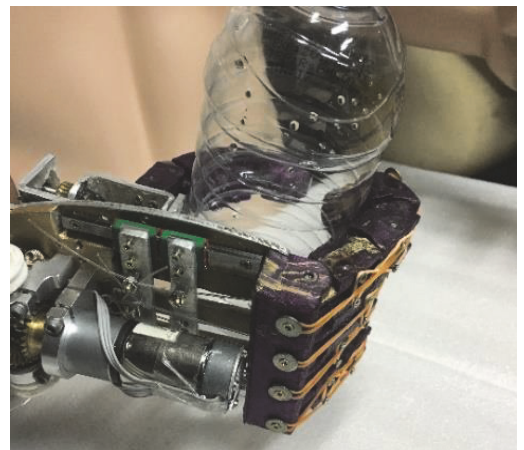

(b)

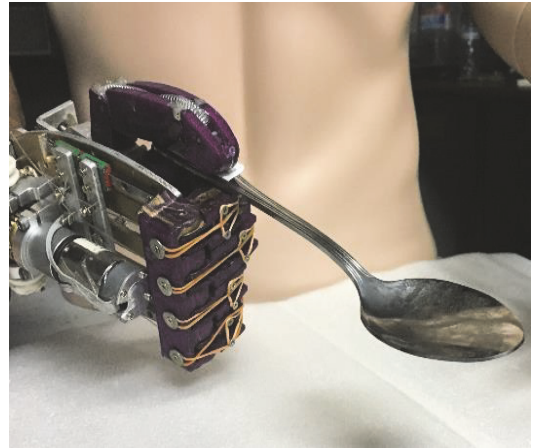

(e)

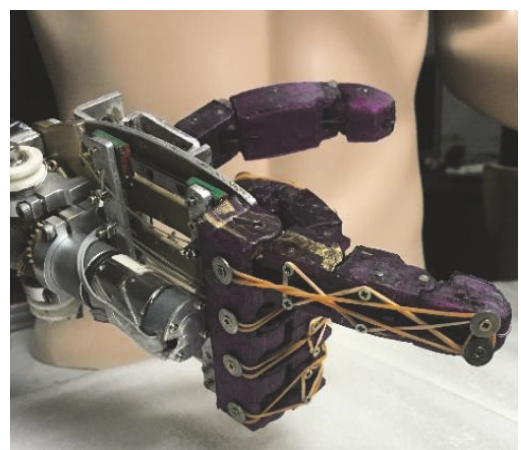

(c)

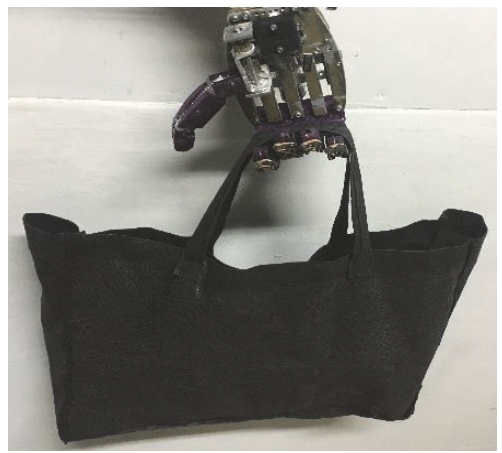

(f)

FIGURE 19: Grasping patterns achieved: (a) rest position; (b) power grip; (c) index point; (d) tip grip; (e) lateral grip; (f) hook grip. 
TABLE 6: Power for executing the desired grasping patterns.

\begin{tabular}{lcccccc}
\hline & \% of total ADL & $\begin{array}{c}\text { \% of achievable } \\
\text { patterns, } f\end{array}$ & $\begin{array}{c}\text { Energy, body } \\
\text { power, } b_{p}(\mathrm{Ws})\end{array}$ & $\begin{array}{c}\text { Energy, electric } \\
\text { power, } e_{p}(\mathrm{Ws})\end{array}$ & $\begin{array}{c}f \times b_{p} \\
(\mathrm{Ws})\end{array}$ & $\begin{array}{c}f \times e_{p} \\
(\mathrm{Ws})\end{array}$ \\
\hline Power grasp & $40 \%$ & $55.4 \%$ & 21.21 & 0 & 11.75 & 0.00 \\
Index finger extension & $13 \%$ & $18.0 \%$ & 0 & 18.79 & 0.00 & 3.38 \\
Tip grasp & $7 \%$ & $9.7 \%$ & 3.53 & 14.83 & 0.34 & 1.44 \\
Lateral grasp & $12 \%$ & $16.6 \%$ & 5.3 & 23.36 & 0.88 & 3.88 \\
Hook grasp & $0.2 \%$ & $0.3 \%$ & 0 & 13.19 & 0.00 & 0.04 \\
\hline Total energy consumption & & & & & 12.97 & 8.74 \\
\% energy consumption & & & & & $59.7 \%$ & $40.3 \%$ \\
\hline
\end{tabular}

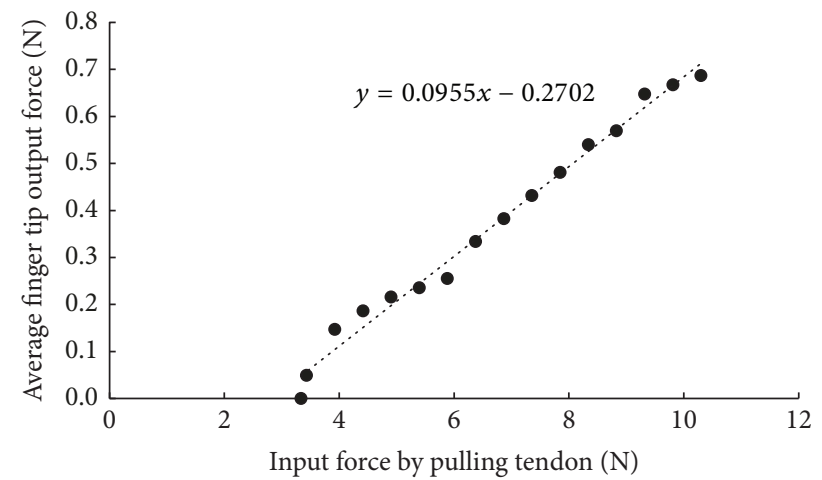

FIGURE 20: Relationship between average fingertip output force and input force.

7.5. Energy Consumption and Power Saving. The energy required for hand preshaping and grasp execution is calculated using the electric current values obtained from the experiments explained in Section 6.4. Table 6 depicts the frequencies and the energy consumed using body power and electric power for each grasping patterns. If the hand is fully operated by the electric power, the total energy consumption of the battery would be the combination of both electric and body energy consumption. According to Table 6, HyPro has the ability of saving $59.7 \%$ of battery usage compared to being fully electrically powered. This considerable amount of battery power saving is mainly because of the power grasp being the most frequent pattern used in ADL. Hence, the hybrid powering concept enables minimizing the battery power consumption which leads to increase battery life. Moreover, the required power from the body movements of the wearer has decreased with the hybrid powering concept. This helps with more comfort and reduces fatigue in repetitive usage of the prosthesis.

Figure 21 shows the energy distribution between body power and battery power when performing each grasp. According to the battery power consumption for different grasps, the power grasp does not require the battery power. Externally powered prostheses are usable when only the power is available [8]. However, the HyPro is capable of performing power grasp even without the electric power. Therefore, it is one of the key advantages of the hybrid powering concept.

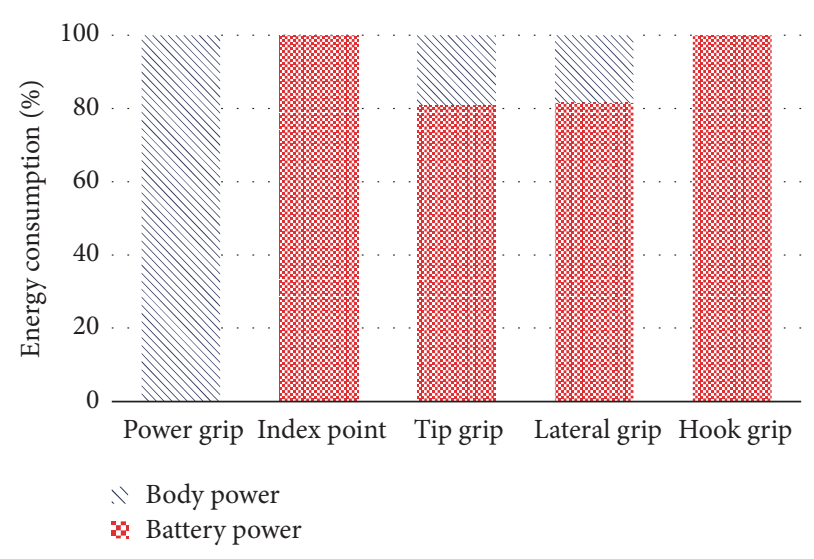

FIGURE 21: Energy distribution of each grasping pattern.

\section{Conclusion}

This paper proposed a hybrid-powered transradial robotic prosthesis, HyPro which can achieve five grasping patterns. The hand unit of HyPro is adopted with hybrid powering concept for grasp function restoration by using both body power and electric power. The proposed hand unit has $15 \mathrm{DoF}$ and actuated only using two electric motors and body power. The wrist unit is capable of achieving two DoF: wrist flexionextension and supination-pronation.

The complexity of controlling the motors is reduced since only two motors are used in this robotic prosthesis. Object grasping can be performed well since the amputee can control the force applied by prosthetic fingers by controlling his body movement from shoulder protraction-retraction. Compared to body-powered prostheses, HyPro has the ability to arrange prosthetic fingers for the desired grasping pattern using electric power. Hence, it does not require to change terminal devices in achieving different grasping patterns as in the conventional body-powered prosthesis. According to the results, the proposed hand unit is capable of saving $60 \%$ of the electric power. Moreover, the prosthetic fingers are capable of adapting to grasp objects like natural human fingers. Besides that, the hybrid design leads to other benefits such as reduction of complexity of controlling actuators and capability of using power grasp while electric power being absent. In particular, a bilateral amputee can use HyPro without the assistance of external party as it does not require 
replacing terminal devices or performing switching action as with most commercially available prostheses.

\section{Conflicts of Interest}

The authors declare that they have no conflicts of interest.

\section{References}

[1] "ISHN.com - the magazine for safety \& health professionals who direct safety \& health programs in high-hazard workplaces," http://www.ishn.com/.

[2] D. S. V. Bandara, R. A. R. C. Gopura, K. T. M. U. Hemapala, and K. Kiguchi, "A multi-DoF anthropomorphic transradial prosthetic arm," in IEEE RAS/EMBS International Conference on Biomedical Robotics and Biomechatronics, pp. 1039-1044, São Paulo, Brazil, 2014.

[3] D. S. V. Bandara, R. A. R. C. Gopura, K. T. M. U. Hemapala, and K. Kiguchi, "Upper extremity prosthetics: current status, challenges and future directions," in International Symposium on Artificial Life and Robotics, pp. 875-880, Beppu, Oita, Japan, 2012.

[4] G. ElKoura and K. Singh, "Handrix: Animating the Human Hand," in SIGGRAPH Symposium on Computer Animation, pp. 110-119, University of Toronto, Canada, 2003.

[5] K. A. Raichle, M. A. Hanley, I. Molton et al., "Prosthesis use in persons with lower- and upper-limb amputation," Journal of Rehabilitation Research and Development, vol. 45, no. 7, pp. 961972, 2008.

[6] F. Cordella, A. L. Ciancio, R. Sacchetti et al., "Literature review on needs of upper limb prosthesis users," Frontiers in Neuroscience, vol. 10, pp. 1-14, 2016.

[7] M. K. Hafshejani, M. Sattari Naeini, and A. Langari, "On the functional limitation in below elbow amputation men using Mechanical and Myoelectric prosthesis via TAPES questionnaire," Life Science Journal, vol. 9, no. 4, pp. 5579-5582, 2012.

[8] C. L. Semasinghe, J. L. B. Prasanna, H. M. Kandamby, R. K. P. S. Ranaweera, D. G. K. Madusanka, and R. A. R. C. Gopura, "Transradial prostheses: Current status and future directions," in Manufacturing \& Industrial Engineering Symposium, pp. 1-7, Colombo, Sri Lanka, 2016.

[9] D. S. Childress and J. N. Billock, "An experiment with the control of a hybrid prosthetic system; electric elbow, bodypowered hook," Bulletin of Prosthetics Research, vol. 10, no. 14, pp. 62-77, 1970.

[10] M. Stobbe, M. R. Dawson, and H. Jacqueline, "Development of a Hybrid Body Powered Transradial. Prosthesis with Myoelectric Switching," http://www.albertahealthservices .ca/grh/Page14577.aspx.

[11] J. C. Becker and N. V. Thakor, "A study of the range of motion of human fingers with application to anthropomorphic designs," IEEE Transactions on Biomedical Engineering, vol. 35, no. 2, pp. 110-117, 1988.

[12] S. Tanrikulu, S. Bekmez, A. Üzümcügil, and G. Leblebicioglu, "Anatomyand Biomechanics of the Wrist and Hand," Sports Injuries, pp. 1-9, 2014.

[13] M. J. Barakat, J. Field, and J. Taylor, "The range of movement of the thumb," HAND, vol. 8, no. 2, pp. 179-182, 2013.
[14] R. A. R. C. Gopura, D. S. V. Bandara, K. Kiguchi, and G. K. I. Mann, "Developments in hardware systems of active upperlimb exoskeleton robots: A review," Robotics and Autonomous Systems, vol. 75, pp. 203-220, 2016.

[15] T. Feix, J. Romero, H.-B. Schmiedmayer, A. M. Dollar, and D. Kragic, "The GRASP taxonomy of human grasp types," IEEE Transactions on Human-Machine Systems, vol. 46, no. 1, pp. 6677, 2016.

[16] L. Wu, G. Carbone, and M. Ceccarelli, "Designing an underactuated mechanism for a 1 active DOF finger operation," Mechanism and Machine Theory, vol. 44, no. 2, pp. 336-348, 2009.

[17] Upper Limb Prosthetics Information, http://www.upperlimbprosthetics.info/index.php?p=1_9_Body-Powered.

[18] L. Resnik, S. L. Klinger, and K. Etter, "The DEKA Arm: Its features, functionality, and evolution during the veterans affairs study to optimize the DEKA Arm," Prosthetics and Orthotics International, vol. 38, no. 6, pp. 492-504, 2014.

[19] E. Kayaoglu, "DLR - Institute of Robotics and Mechatronics Data sheet of DLR Hand II," http://www.dlr.de/rmc/rm/en/ desktopdefault.aspx/tabid-3802/6102_read-8922/.

[20] H. Liu, K. Wu, P. Meusel et al., "Multisensory five-finger dexterous hand: The DLR/HIT hand II," in Proceedings of the IEEE/RSJ International Conference on Intelligent Robots and Systems (IROS '08), pp. 3692-3697, September 2008.

[21] J. L. Pons, E. Rocon, R. Ceres et al., "The MANUS-HAND dextrous robotics upper limb prosthesis: mechanical and manipulation aspects," Autonomous Robots, vol. 16, no. 2, pp. 143-163, 2004.

[22] A. Polhemus, B. Doherty, K. Mackiw, R. Patel, and M. Paliwal, "uGrip II: A Novel Functional Hybrid Prosthetic Hand Design," in Proceedings of the 39th Annual Northeast Bioengineering Conference, (NEBEC '13), pp. 303-304, USA, April 2013.

[23] Touchbionics.com, "i-limb ultra - Touch Bionics," http://www. .touchbionics.com/products/active-prostheses/i-limb-ultra.

[24] Ottobock.co.uk, "Solution overview upper limb prosthetics Ottobock UK," https://www.ottobock.co.uk/prosthetics/upper_ limbs_prosthetics/product-systems/.

[25] D.-P. Yang, J.-D. Zhao, Y.-K. Gu et al., "An anthropomorphic robot hand developed based on underactuated mechanism and controlled by emg signals," Journal of Bionic Engineering, vol. 6, no. 3, pp. 255-263, 2009.

[26] Hosmer.com, “Hosmer Hooks," http://hosmer.com/products/ hooks/index.html.

[27] Bebionic.com, "Life changing myoelectric hand packed with the latest technology - Bebionic," http://bebionic.com/the_hand.

[28] J. Hamill and K. M. Knutzen, Biomechanical Basis of Human Movement, Lippincott Williams \& Wilkins, Pa, USA, 2006.

[29] D. S. V. Bandara, R. A. R. C. Gopura, G. Kajanthan, M. Brunthavan, and H. I. M. M. Abeynayake, "An under-actuated mechanism for a robotic finger," in Proceedings of the 4th Annual IEEE International Conference on Cyber Technology in Automation, Control and Intelligent Systems, (IEEE-CYBER '14), pp. 407-412, China, June 2014.

[30] J. Z. Zheng, S. De La Rosa, and A. M. Dollar, "An investigation of grasp type and frequency in daily household and machine shop tasks," in Proceedings of the 2011 IEEE International Conference on Robotics and Automation, (ICRA '11), pp. 4169-4175, China, May 2011. 
[31] G. I. Bain, N. Polites, B. G. Higgs, R. J. Heptinstall, and A. M. McGrath, "The functional range of motion of the finger joints," Journal of Hand Surgery (European Volume), vol. 40, no. 4, pp. 406-411, 2015.

[32] C. Pylatiuk, S. Schulz, and L. Döderlein, "Results of an internet survey of myoelectric prosthetic hand users," Prosthetics and Orthotics International, vol. 31, no. 4, pp. 362-370, 2007.

[33] P. Rea, "On the design of underactuated finger mechanisms for robotic hands," in Advances in Mechatronics, H. MartinezAlfaro, Ed., 2011.

[34] Anthropometry and Biomechanics, "Msis.jsc.nasa.gov," https:// msis.jsc.nasa.gov/sections/section03.htm. 


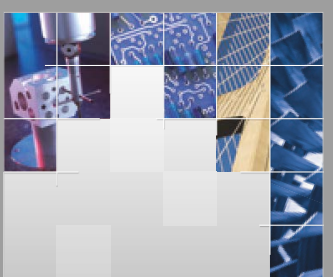

\section{Enfincering}
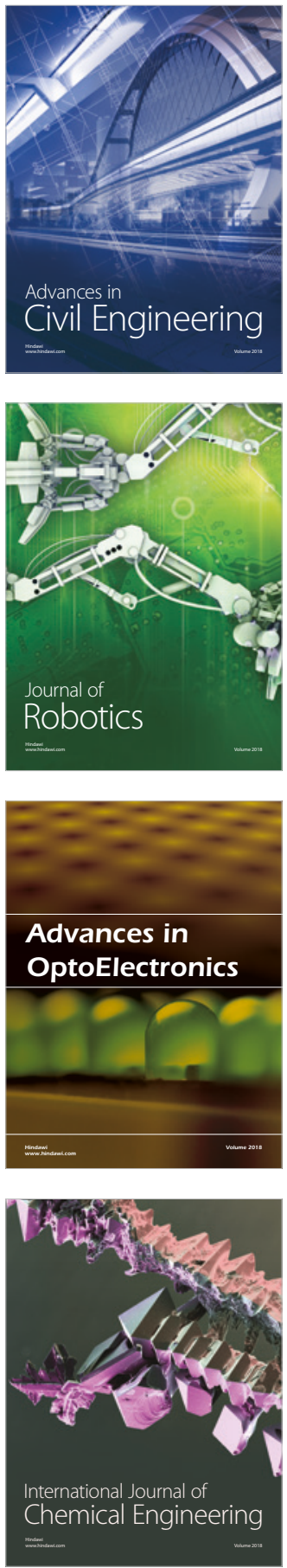

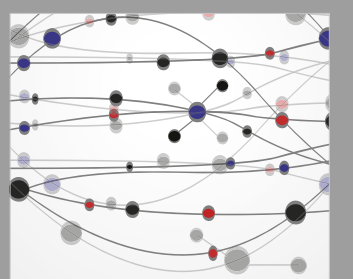

\section{Rotating \\ Machinery}

The Scientific World Journal

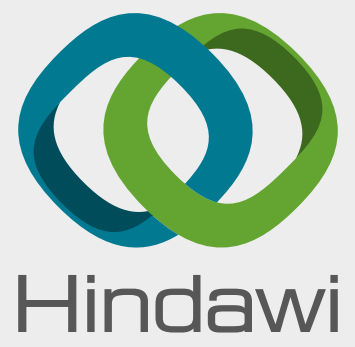

Submit your manuscripts at

www.hindawi.com
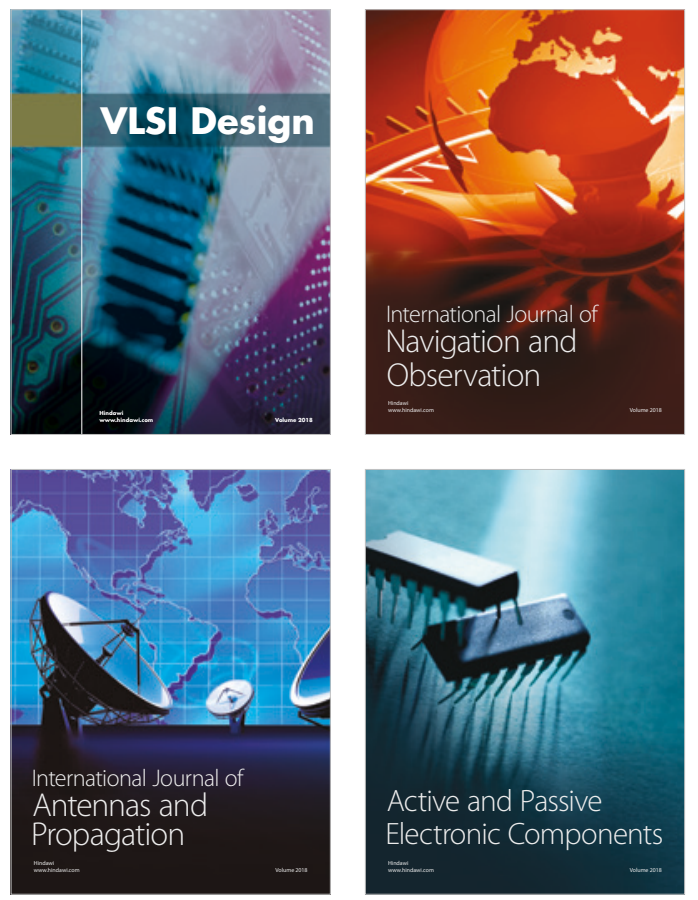
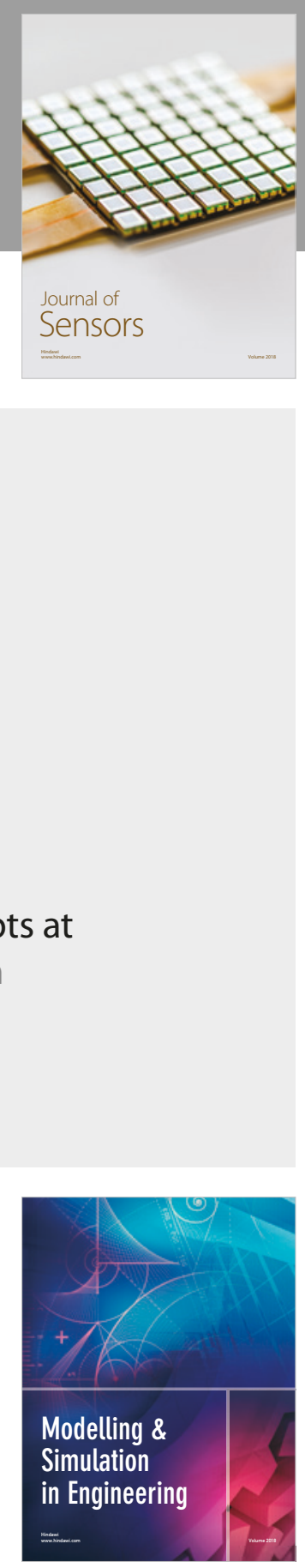

\section{Advances \\ Multimedia}
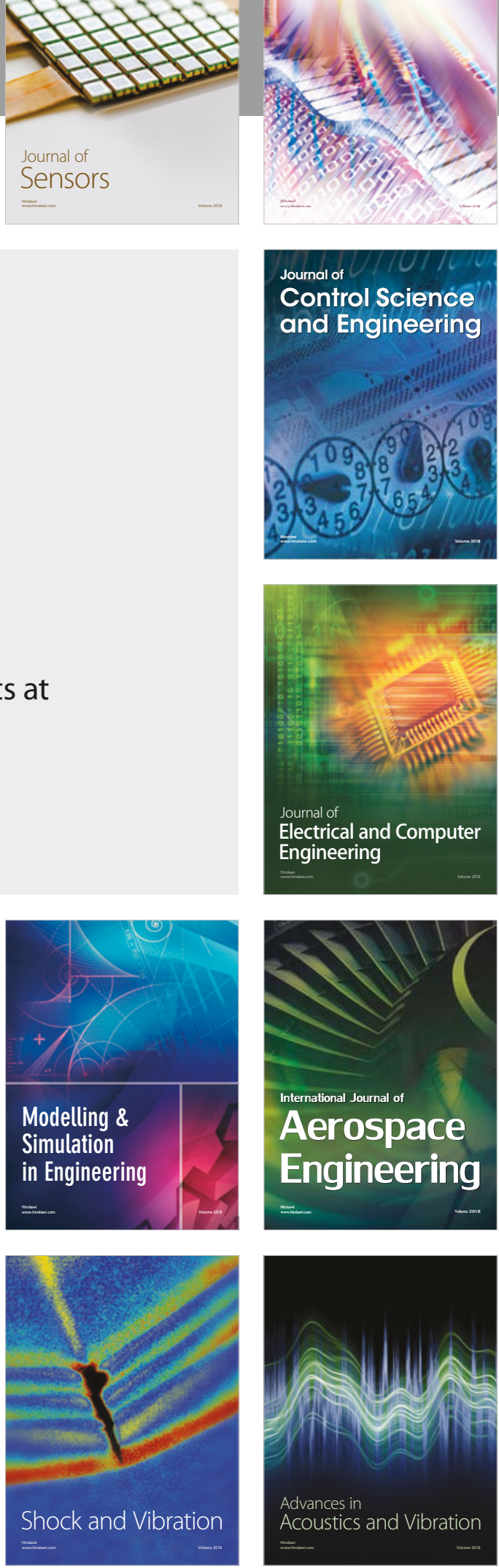